\title{
Air Quality Forecast Based on Neural Network
}

\author{
Shengping Zhao ${ }^{1}$ and Jingrui $\mathrm{Li}^{2}$ \\ ${ }^{1}$ Yuxi Normal University, Yuxi, 653100, China; \\ ${ }^{2}$ Yunnan Power Co., Ltd. Honghe Power Supply Bureau, Mengzi, 661100, China
}

Keywords: BP neural network; air quality forecast; historical data; improved algorithm

\begin{abstract}
There is a wide public concern on air quality. The precise forecast of air quality is of significant importance for production, life and atmospheric control. This study takes the air quality in Yuxi City as the research object. And the Pearson correlation coefficients between 6 conventional meteorological factors, the concentration of 6 pollutants and AQI are analyzed. AQI value is forecasted based on the values of previous $n$ days. Air quality forecast can generally be done through BP network, but there are problems like overfitting. Therefore, some improvement is made in BP network. Base on the analysis of the three years AQI tendency chart, it is proposed in the study to add historical contemporaneous meteorological data to the Input. And it is proved that this addition improves the forecast precision. Besides, the study proposes the ideas about improving excitation function and using cascade network for the further improvement of model forecast performance.
\end{abstract}

Over the recent years, the total emission of air pollutants in our country is decreasing year by year as there is more public concern and attention on air quality. The air quality in some once seriously polluted cities is also getting better, but the proportion of cities where the air quality reaches Level 2 Standard is decreasing. Air pollution with particulate matter, sulfur dioxide and nitrogen oxides as the main pollutants is getting worse[1]. This study analyzes the air quality in Yuxi City and finds that the air quality there is in a tendency of slow degradation.

Precise air quality modeling forecast can not only provide data support for effective atmospheric control and reduce control cost dramatically, but also provide reference to company and society behavior. Air quality is a non-linear problem influenced by multiple factors. Neural network can do a good approximation on non-linear problem by studying the input samples so as to make precise air quality forecast for the specific location or area.

Neural network is widely used in air quality forecast. And BP neural network is the most typical feedforward network with the ability to approximate any non-linear relationship, but there are shortcomings such as slow convergence and being easily getting into local minimum[2. 3]. Xie Chao and several other researchers[4] establish BP, Elman, T-S fuzzy and wavelet transform neural networks to do air quality forecast and get the conclusions that Elman neural network has a good dynamic feedback ability and better modeling forecast precision and universality. Therefore, it is better to use dynamic neural network for air quality forecast. Ren Ganghong and several other researchers[5] use cascade neural network together with BP and RBF neural networks for rainfall forecast. The cooperation between these neural networks allows for higher forecast precision. However, most of the previous air quality forecast models neglect the influence of the historical air quality data on the present air quality.

\section{Establishment of neural network model}

BP neural network mainly features forward transmission of information and reverse transmission of error with high reliability and fault tolerance, strong generalization ability and simple structure. Some improvement is made on the basis of BP network and momentum factor and adaptive modified learning rate are added to improve network training efficiency and model adaptability[6]. Meanwhile, historical meteorological data of the previous 1 or 2 years is added to the Input so as to 
increase forecast precision.

AQI, short for Air Quality Index, is a dimensionless index for quantitative description of air quality and a parameter in daily air quality report. It shows the degree of air cleanness or pollution and the effect on human health. There are also IAQI for individual pollutant description. The main pollutants involved in air quality evaluation include fine particulate matter, inhalable particulate matter, sulfur dioxide, nitrogen dioxide, ozone, carbon monoxide.

Correlation analysis is carried out on the basis of monitoring data of conventional meteorological factors and pollutant concentration of year 2015 in Yuxi City (Air quality data is from the China air quality online monitoring and analysis platform: https://www.aqistudy.cn/ ). Pearson correlation coefficients are used to respectively calculate the relations between AQI and parameters of 6 main pollutants (PM2.5, PM10, $\mathrm{So}_{2}, \mathrm{No}_{2}$, etc.) and 6 meteorological factors (relative humidity, accumulated precipitation, sunshine hours, average wind speed, maximum wind speed, average temperature), providing data support for the following analysis and parameter setting[7-8].

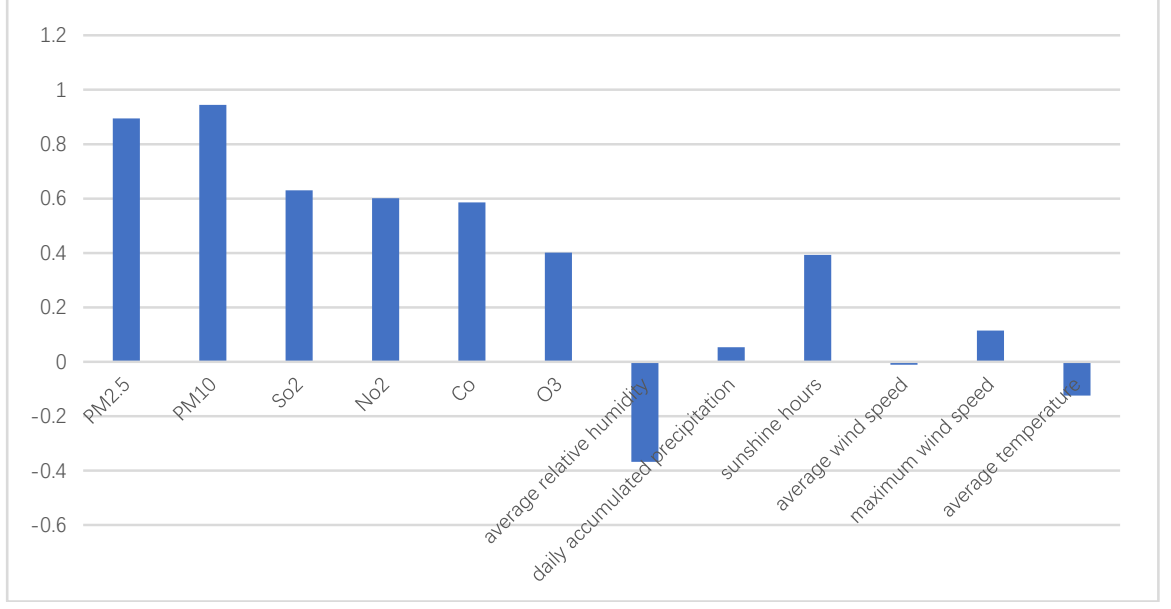

Figure 1. Pearson correlation coefficients between AQI and12 pollutants parameters and meteorological factors

\section{Analysis of neural network forecast}

\subsection{BP neural network forecast}

The Pearson correlation coefficients between AQI and the parameters of 6 pollutant like PM2.5 are high as these 6 parameters are the main indicators in AQI evaluation. The Pearson correlation coefficients between AQI and 6 meteorological factors like relative humidity are lower, among which average relative humidity -0.3680 and sunshine hours 0.3931 are relatively higher, and average wind speed -0.011 is the lowest value. Therefore, the average wind speed factor can be removed. The 11 parameters of $\mathrm{PM} 2.5, \mathrm{PM} 10, \mathrm{So}_{2}, \mathrm{No}_{2}, \mathrm{CO}, \mathrm{O}_{3}$, relative humidity, accumulated precipitation, sunshine hours, maximum wind speed, average temperature can be used as training data for network.

The pollutants parameters and meteorological information of the previous 7 days are the input data of the network. The output is AQI value. The total amount of samples is 1000, among which at least 900 are randomly selected to serve as the training samples and 51 to serve as test samples. The neural network set up in this way has 77 inputs in total and 1 output. The hidden layer node is calculated with the empirical formula and step-up procedure. The hidden layer node number is 8 . The forecast curve is as shown in Figure 1.

The MAE(Mean Absolute Error) of the forecast result is 0.390, which is a relatively large deviation. And there is overfitting in the training process, as shown in figure 2. The linear regression is unsatisfactory with Validation. $R=0.49$ and Test. $R=0.6$. Meanwhile, there is no sufficient training steps, which negatively affects the final forecast. 


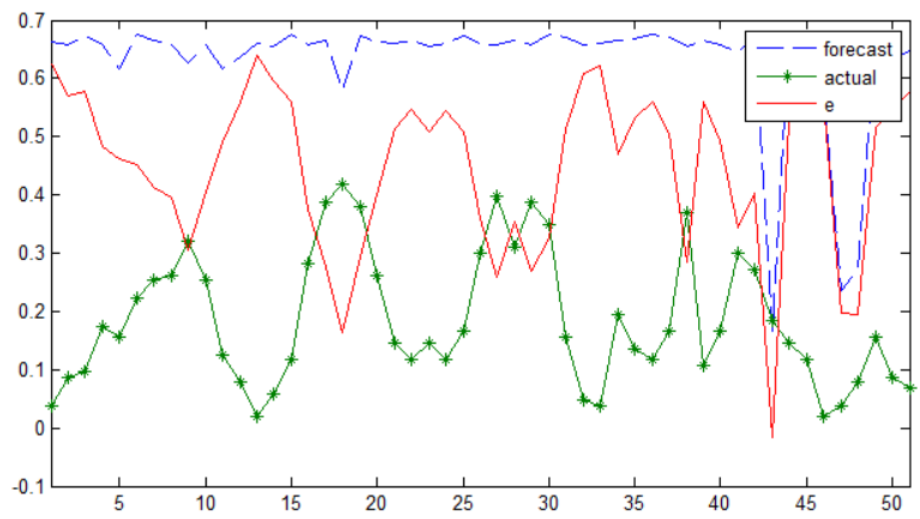

Figure 2. AQI forecast curve graph

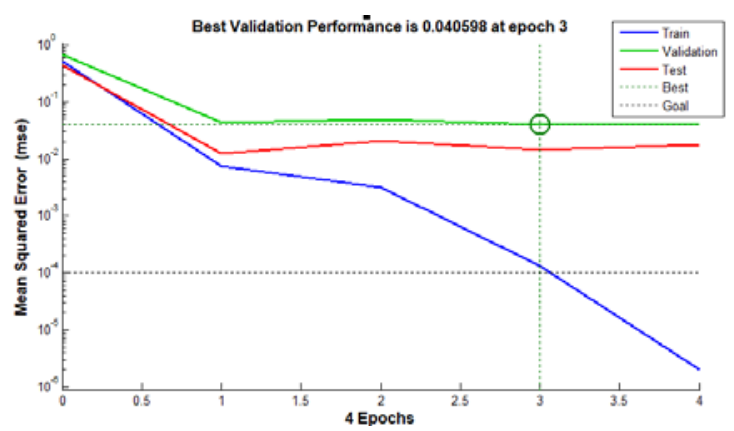

Figure 3. MSE curve graph

\subsection{Network forecast improvement}

Gradient descent method is used in BP neural network training to correct weight and threshold. The threshold and weight of the network are corrected from the negative gradient direction of the forecast error. The learning and accumulation of historical experience are neglected in the process of training when the gradient descent method is applied. What's more, the convergence of the learning process is slow. Therefore, the addition of momentum should be considered to improve learning effect.

Learning rate means the speed at which the information is accumulated in the network. The speed at which the neural network reaches (and whether it can reach) the best and most advantageous position for a given output is determined by learning rate. In the original stochastic gradient descent (SGD), learning rate is not influenced by the shape of the error gradient, because the global learning rate which is not related with error gradient is used. However, the original SGD update rule can be modified and the neural network learning rate can automatically adjust learning. The adaptive learning rate method is proposed. Set the multiple of increasing learning rate to 1.05 and the multiple of decreasing learning rate to 0.8 . This results in the optimization of forecast with MAE reaching 0.125 and less overfitting. There is neither satisfactory linear regression nor obvious optimization. The forecast curve is as shown in Figure 3.

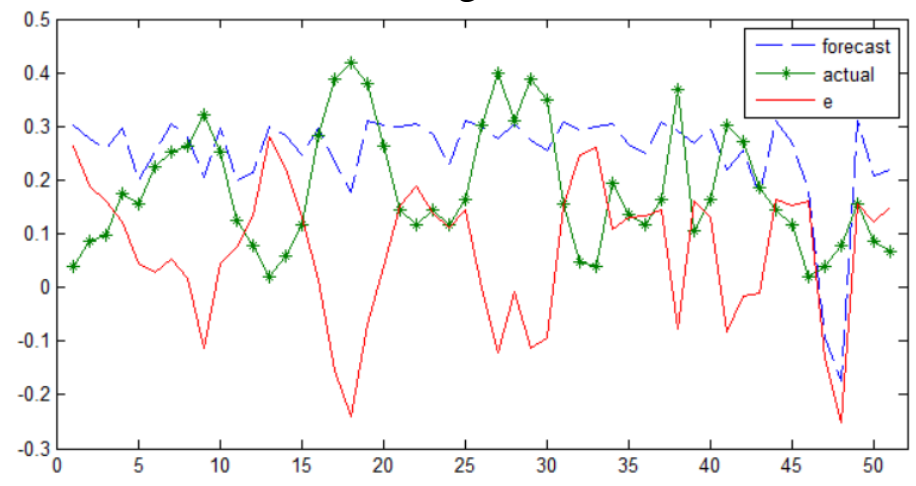

Figure 4. forecast curve obtained after momentum and adaptive learning rate are used 
To improve the forecast precision, analyze the influence of taking pollutants parameters and meteorological information of previous $n$ days as input data on the forecast results. When $n$ is set in the range of 1-30, the quantity of optimum forecast results among the 20 forecast results is as shown in Figure 4. The effect is better in case that $\mathrm{n}$ is in the range of 1-10, but without decisive advantage. So, the value of $n$ has little influence on forecast results. The value of $n$ is set at 4 in the following forecast.

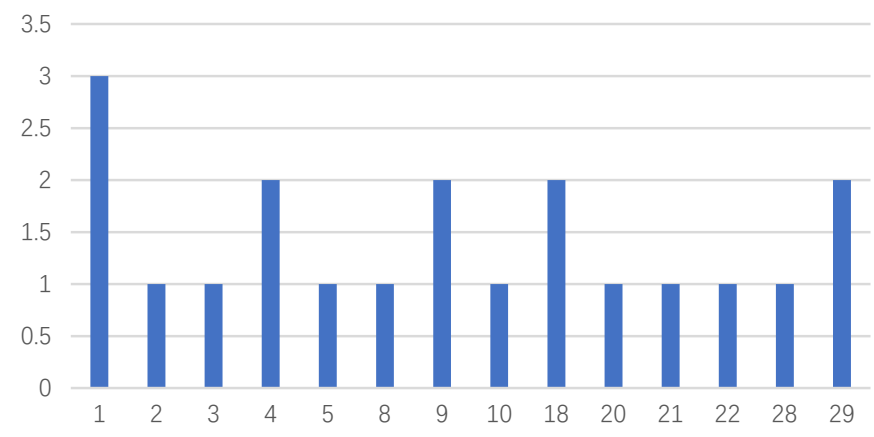

Figure 5. number of optimum forecast results when $n=1-30$

\subsection{Analysis on the influence of historical meteorological data}

Meteorological information features continuity and regularity. AQI forecast is also affected by historical contemporaneous data. Figure 5 is the AQI curves in the year 2014, 2015 and 2016. It can be concluded that the air quality has the same change tendency throughout the year despite some difference in values due to the influence of actual weather condition and some individual factor. So, not only the influence of previous $n$ days but also the influence of historical AQI, pollutant parameters and meteorological information of previous 1 or 2 years or even longer ago should be taken into account.

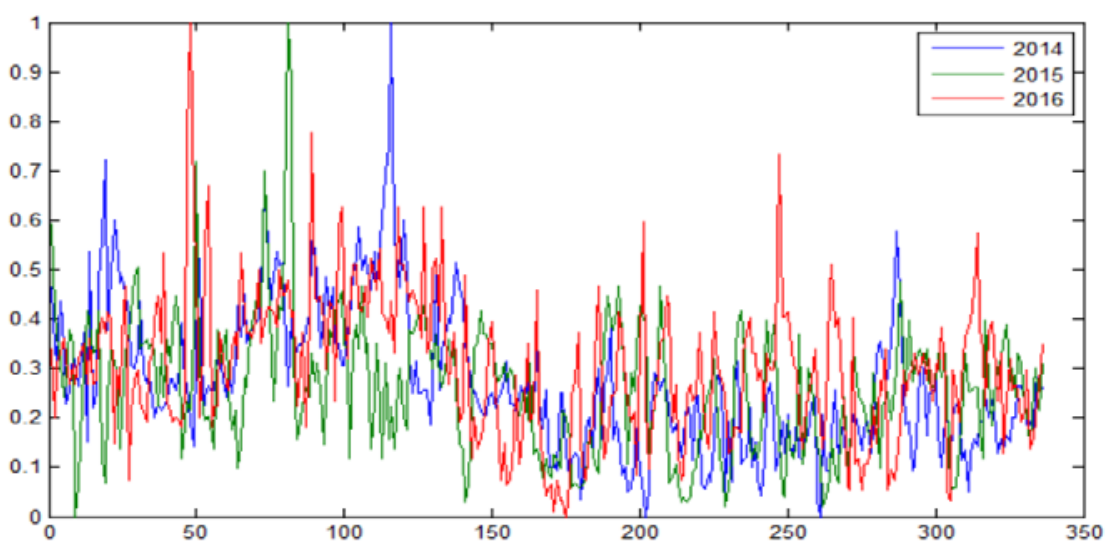

Figure 6. AQI curves in the year 2014, 2015, 2016

Set a new neural network with pollutant parameters and meteorological information of previous 4 days and the historical data of this day in the year 2014 and 2015 being the input, and AQI being the output. The total amount of samples is 1000, among which at least 900 are randomly selected to serve as the training samples and 51 to serve as test samples. The neural network set up in this way has 68 inputs in total and 1 output. The result forecast curve is as shown in Figure 6. The MAE of the forecast result is 0.095 . Validation. $R=0.8$, Test. $R=0.8$.

A comprehensive analysis of the above neural network forecast results can lead to the conclusion that the model with the addition of historical contemporaneous data shows better precision in forecast. MAE of 51 test values is improved by $70 \%$ with better linear regression. See Chart 1 for the specific parameters. 


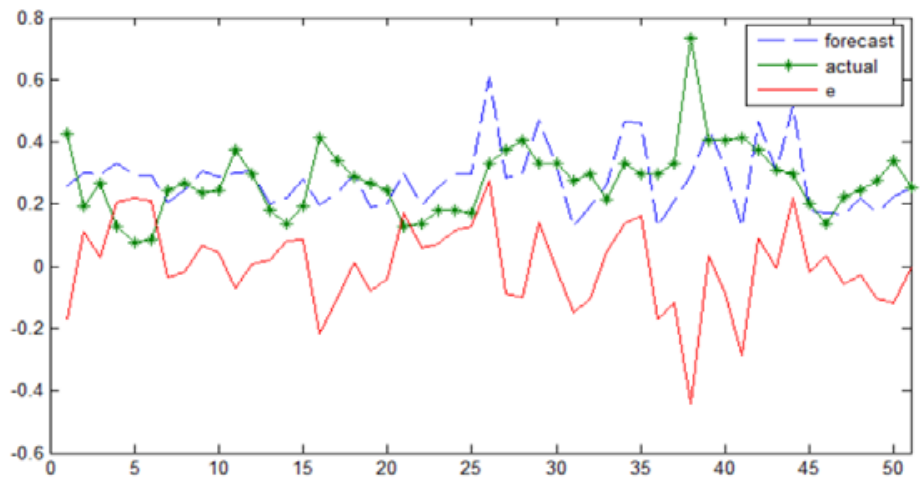

Figure 7. forecast curve after the historical data of the year 2014 and 2015 is input

Table 1. Comparison of data of 3 networks

\begin{tabular}{cccc}
\hline Item & $\begin{array}{c}\text { Basic } \\
\text { network }\end{array}$ & $\begin{array}{c}\text { Improved neural } \\
\text { network }\end{array}$ & $\begin{array}{c}\text { Network with the } \\
\text { addition of historical } \\
\text { data }\end{array}$ \\
\hline Input node & 77 & 77 & 68 \\
Hidden layer node & 8 & 8 & 6 \\
$\begin{array}{c}\text { Linear regression } \\
\text { Mean absolute }\end{array}$ & 0.6 & 0.65 & 0.8 \\
error(MAE) & 0.39 & 0.125 & 0.095 \\
\hline
\end{tabular}

\section{Conclusion}

The traditional BP network can do the basic air quality forecast, but the forecast precision is not ideal with shortcomings such as getting into local minimum and overfitting. The network performance and model learning ability can be improved by adding momentum factor and adaptive learning rate, etc. It is found by forecast result comparison that the forecast precision can be greatly improved with the addition of historical data.

Besides, several problems encountered in the modeling process deserve future research and study: 1) If sigmoid function is selected for hidden layer, there would be problems such as early loss of the derivative and insufficient training steps. So, it is necessary to select and design some excitation function more suitable, and to improve network performance by adding the loss function of cross entropy. 2) Cascade neural network has a wide application with good forecast ability, but it is seldom used in air quality forecast and needs further simulated test. 3) The related economic factor should also be considered for forecast model[9].

\section{Acknowledgements}

Science and Technology Planning Project (Youth Project 2018FD094), Science and Technology Department, Yunnan Province;

Science and Technology Planning Project (Youth Project 2017FD162), Science and Technology Department, Yunnan Province;

\section{References}

[1] Liang Yinshuang, Sun Zhenying, Shen Qixia. The Analysis on Air Quality Variation Trends and Influence Factors — Take Beijing City as an Example [J]. Journal of Zhongzhou University 2015(05)

[2] Reich S. L. , Gomez D.R. , DawidowskiL. E. Artificialneural Network for the Identification of Unknown Air Pollution Sources Atmospheric Environment. 1999, 33(18):3045-3052

[3] Zhao Liangdong, Yin Runyuan, Wu Qian. An Anomaly Detection Method Based on Improved 
BP Neural Network [J]. Microcomputer Information. 20101(28)

[4] Xie Chao, Ma Mintao, Yu Xiaoxiao. Application of Multiple Neural Networks in Air Quality Forecast in Cities of Western and Northern China [J]. Journal of Environmental Engineering. 2015(12):6006-6008.

[5] Ren Ganghong, Du Kun, Zhou Ming, Liu Niandong, Zhang Jin. Annual Rainfall Forecast Based on Cascade Neural Network [J].Civil and Environment Engineering. [5]2016(26):137-141.

[6] Zhang Guoyi, Hu Zheng. Analysis on the Improvement of BP Neural Network Model and Stability[J]. Journal of Central South University (Nature and Science Version).2011(01)

[7] Wang Jian, Hu Xiaomin, Zheng Longxi, Liu Zhenshan. Research on the Method of Atmospheric Pollution Forecast Based on BP Model [J]. Environment and Science Research. 2002(05)

[8] Zhang Yiwen, Ao Xiqin, Shi Peijun, Guo Aodong, Fei Jiulong, Chen Jiali. BP Neural Network PM2.5 Forecast Model Based on Pearson Correlation Index [J]. Journal of Qingdao University (Nature and Science Version).2017(02)

[9] Cui Hua, Bai Jie, Bi Xin, Huang Libo. Deep Neural Network with Multiple Cascaded Output Layers [J]. Journal of Tongji University (Nature and Science Version).2017(S1) 